\title{
Mathematikausbildung in der Diskussion: Ist die Kompetenzorientierung wirklich schuld?
}

\author{
Ein Blick in die Schulpraxis
}

\author{
Brigitte Lutz-Westphal
}

\begin{abstract}
In der aktuellen Diskussion um die Mathematikausbildung gibt es zahlreiche Stimmen, die behaupten, die in den Bildungsstandards geforderte Kompetenzorientierung sei schuld an den angeprangerten Problemen. Diese Stimmen kommen mehrheitlich aus Fachhochschulen oder Universitäten. Wir haben daher einige Lehrerinnen und Lehrer gebeten, uns für unsere neue Serie der „O-Töne aus der Schulpraxis“ ihre Gedanken zur folgenden Frage aufzuschreiben: Kompetenzorientierung in der Schule - Fluch oder Segen?
\end{abstract}

\begin{abstract}
Sämtliche zu dieser Frage bei uns eingegangenen Texte drucken wir im Folgenden ab. Alle Autor/innen befürworten die Kompetenzorientierung, sehen sie als Gewinn und wichtigen Bestandteil einer guten schulischen Mathematikausbildung. Der Blick in die Schulpraxis und das Gespräch mit denjenigen, die den alltäglichen Unterricht gestalten, scheint hier also ein ganz anderes Bild zu ergeben als dasjenige, das momentan von einigen Diskutant/innen gezeichnet wird. Lassen wir uns also aus der Praxis berichten.
\end{abstract}

\section{Lehrer, Stadtteilschule Hamburg}

Als Lehrer an einer Hamburger Stadtteilschule, die auch einen gymnasialen Zweig in der Mittelstufe und eine gemeinsame Studienstufe umfasst, ist es meine jahrelange zentrale Erfahrung, dass Kompetenzorientierung der einzig richtige Weg im Unterricht ist. Denn abgesehen von einigen wenigen SchülerInnen, die von der inneren Logik und Systematik des Faches fasziniert sind, können wir die große Mehrzahl der SchülerInnen - auch der Studienstufe - nur für den Mathematikunterricht gewinnen, wenn sie die Bedeutung des Faches für ihre eigene Umwelt und ihr Handeln darin erkennen. D. h. sie benötigen zuvörderst mathematische Kompetenzen, durch die sie dazu in die Lage versetzt werden. Einzig die Entdeckung, wie hilfreich Mathematik für die eigene Lebenswelt ist, gewinnt die Lernenden dafür, sich auch mit der Mathematik selbst intensiver auseinanderzusetzen. So sind auch Kompetenzund Fachorientierung gar keine Gegensätze, sondern erst durch den Schwerpunkt, den wir auf das erste legen, wird auch das zweite möglich.

\section{Lehrerin, Gymnasium Berlin}

Die Kompetenzorientierung des Mathematikunterrichts ist in jedem Fall ein Gewinn. So findet die Lehrkraft im Rahmenlehrplan durch die Formulierung der zu erwerbenden Kompetenzen konkretere Hinweise zur Gestaltung des Lehr- und Lernprozesses. Die SchülerInnen sind durch die konkrete Benennung der Kompetenzen besser in der Lage, eigene Stärken und Schwächen zu erkennen und können ihren eigenen Lernprozess besser mit gestalten. Wünschenswert wäre jedoch, wenn für die Entwicklung dieser Kompetenzen im Schulalltag mehr Zeit und Raum bliebe, wozu meines Erachtens in einigen Niveaustufen des Rahmenlehrplans eine weitere Ausdünnung der Lerninhalte erfolgen müsste.

\section{Lehrer i. R., Gymnasium Baden-Württemberg}

Wenn Kompetenzorientierung, wie viele HochschulmathematikerInnen glauben, ein Fluch ist, was ist dann der Segen? Inhaltsorientierung?

Stricken ohne Wolle ist sinnlos. Ebenso sinnlos ist die Vermittlung mathematischer Kompetenzen ohne Inhalte. Aber Wolle zu erwerben, ohne mit ihr zu stricken, ist eine Fehlinvestition. Mathematische Inhalte zu erwerben, ohne mit diesen produktiv zu arbeiten, ist auch eine Fehlinvestition.

Die Frage, ob Kompetenz- oder Inhaltsorientierung wichtiger ist, ist genauso unsinnig wie die Frage, ob für den Flächeninhalt eines Rechtecks die Länge oder die Breite wichtiger ist. Fazit: Kompetenzorientierung ist ein Segen, aber nur wenn sie mit der Vermittlung zentraler Inhalte verknüpft ist, Kompetenzorientierung ohne Inhalte ist ein Fluch. Dass die mathematische Qualität von Studienanfängern rückläufig ist, kann nicht bestritten werden. Dafür sollte aber nicht die Kompetenzorientierung als Sündenbock genommen werden. Es gibt wichtigere Ursachen: Starke Reduzierung der Stundenzahlen für Mathematik, Abschaffung von Leistungskursen, immer höhere Übergangsquote ans Gymnasium, Image der Mathematik in der Schule und in der Öffentlichkeit, ...

\section{Referendarin, Gymnasium Hamburg}

Im Prinzip bin ich absolut pro Kompetenzorientierung, da ich sie immer als gewinnbringend wahrgenommen habe. Die SchülerInnen haben meist mehr Spaß daran und sind sehr motiviert. Doch leider wird es immer wieder problematisch, wenn es in Richtung der Leistungsüberprüfun- 


\section{Apropos „Mathematikausbildung - die Diskussion braucht mehr Perspektiven!“}

Vorschläge für einen multiperspektivischen Blick Die seit 2014 in den Mitteilungen stattfindende Diskussion um den Mathematikunterricht an Schulen verläuft tatsächlich in zu enger Bahn, begleitet vielleicht von einem gewissen Fachegoismus. Anzeichen hierfür sind die Forderungen nach

- einer Erhöhung der Stundenzahlen für den Mathematikunterricht, ohne zu sagen, zu Lasten welcher anderer Fächer dies geschehen soll;

- der Studierfähigkeit von Abiturienten für ein WiMINT-Fach und dabei die Studierfähigkeit für andere Fachrichtungen (Jura, Medizin, Kunst, Sprachen etc.) nicht zu berücksichtigen;

- der Rückkehr von G8 zu G9, obwohl in manchen anderen Ländern (und bis 1990 in der DDR) zwölf Jahre Schulunterricht für ein Studium an Universitäten ausreich(t)en.

\section{"Mehr Perspektiven“}

Die im Folgenden genannten Aufgaben hängen voneinander $\mathrm{ab}$, und bei ihrer Bearbeitung (wohl kaum: Bewältigung) sind alle Gruppen gefragt.

Die Gesellschaft, letztlich also Parlamente und Kultusbürokratien, bestimmen über den Bildungsauftrag von Schulen und was deren Abschlusszeugnisse bestätigen sollen. Deckt Studierfähigkeit schon alles ab? Auch bei Absolventen, die (zunächst) nicht studieren wollen?

Erziehungswissenschaft und alle Fächer (nicht nur die Fachdidaktiken) müssen gemeinsam den Bildungsauftrag von Schulen mit Inhalten füllen. Dabei ist nachrangig, ob man diese Inhalte durch Kompetenzen beschreibt oder durch Qualifikationen (wie während der 1970er Jahre) oder ,altdeutsch“ durch Wissen, Fertigkeiten, Fähigkeiten und Einstellungen.
Die Schulpraktiker entscheiden gemeinsam, welche Wünsche und Forderungen sie überhaupt erfüllen wollen und können (und zwar beim alltäglichen Unterrichten), ob und was für eine „Reife“ ein Abschlusszeugnis bescheinigen möchte.

Die Lehrenden an Hochschulen beschreiben gemeinsam, was allgemeine Studierfähigkeit (für alle Studienfächer) sein soll und was außerdem zum Studium einer speziellen Fachrichtung vorteilhaft ist.

Ziemlich sicher kommen bei solchen Aushandlungen zunächst viel zu umfangreiche ,kleinste gemeinsame Nenner" (gemeint im Sinne der kgV der Teilbarkeitslehre) heraus. Dann muss jede Gruppe sich bescheiden und etwas von ihren Wünschen und Forderungen zurücknehmen. (Das gilt natürlich auch für die Anzahl der Unterrichtsstunden jedes Schulfaches.) Schließlich: „Auf den Lehrer kommt es an!“ Und auf manch Anderes. Das heißt:

- Man darf nicht nur ideale Verhältnisse unterstellen.

So ist z. B. nicht jeder Lehrer so gut, wie man selbst gerne wäre.

- Jeder Lehrer hat eine eigene Persönlichkeit, seine Lerngruppen unterscheiden sich voneinander, sonstige „Rand“-Bedingungen sind oft „zentral“ mitbestimmend. Daher verlangt jeder Unterricht vom Lehrer immer wieder Eigenständigkeit. Vorbildliches Unterrichten anderer kann nur zum Nachdenken über eigenes Tun anregen, sollte aber nicht zum bloßen Nachmachen dienen.

Lothar Profke

Prof. (i. R.) Dr. Lothar Profke, Justus-Liebig-Universität Gießen, Karl-Glöckner-Straße 21 C, 35394 Gießen lothar.profke@math.uni-giessen.de gen geht. Zumindest an meiner Schule ist es „Brauch“, dass alle Kolleginnen und Kollegen dieselben Klassenarbeit innerhalb eines Jahrgangs schreiben. [... ] Ich bin ja immer schon ganz froh, wenn die SchülerInnen auch mal etwas schreiben/beschreiben dürfen und nicht nur stumpf Dinge berechnen. Der Traum, mal eine Modellierungsaufgabe in einer Klassenarbeit abzufragen, steht da noch ganz weit entfernt in den Sternen. [...] Aber sollten wir die SchülerInnen nicht eigentlich auch auf das Lösen von Problemen vorbereiten? Das, was sie später auch im Job können sollten? Wichtig ist, dass die SchülerInnen in allen Bereichen des MU ein Gleichgewicht zwischen „Rechnen“ und „Kompetenzorientierung“ kennenlernen, weder das eine, noch das andere sollte überwiegen.

\section{Lehrer, Grundschule Berlin}

Es ist das Schicksal bildungspolitischer Reformen, von den einen als Segen und von den andern als Fluch dargestellt zu werden. Hier geht es der Kompetenzorientierung nicht anders als der Lernzielorientierung, der vorhergehenden, größeren pädagogischen Reform der zweiten Hälfte des letzten Jahrhunderts.

Von Segen für Schule und Gesellschaft sprechen BefürworterInnen der Kompetenzorientierung, wenn sie glauben, dass damit erfolgreicher Unterricht, eine bessere Steuerung von Bildung und ein erfolgreicheres Abschneiden bei nationalen und internationalen Vergleichstests garantiert sei. Von Fluch sprechen die GegnerInnen der Reform, wenn sie Kompetenzorientierung als Gängelung der Lehre durch die Politik, als Versuch, Bildung zu ökonomisieren und auf „Output“ hin zu optimieren oder gar von „Kapitalisierung der Bildung“ sprechen.

Die Wahrheit liegt irgendwo dazwischen und abseits der mit dieser Reform einhergehenden Polemik. Denn Kompetenzorientierung bedeutet nach wie vor mathematische Bildung und Wissensvermittlung, „Studium“ im Sinne mathematischer Auseinandersetzung - aber eben nicht nur. Zudem: auch kompetenzorientierter Mathema- 
tikunterricht kann scheitern. Gerade die Einsicht, dass mathematische Kompetenzen nur bedingt lehrbar sind und ihr Erwerb wesentlich von den AkteurInnen (also zunächst den Lehrenden UND Lernenden) abhängt, legt Scheitern sogar sehr nahe.

Wie wirkt sich Kompetenzorientierung im Mathematikunterricht aus? Wie äußert sich in Mathefachkonferenzen und Klassenräumen Fluch und Segen der Kompetenzorientierung? Mathematiklehrende „fluchen“ über die Kompetenzorientierung, wenn sie neben dem Unterricht mit seinen Verpflichtungen ein schulinternes und natürlich kompetenzorientiertes Mathematikcurriculum schreiben sollen. Obwohl gemeinhin Konsens besteht, dass Kompetenzorientierung eine Verbesserung des Mathematikunterrichts bedeutet, fehlt es für die Umsetzung an professioneller, fachlicher Begleitung und Unterstützung und - fast noch wichtiger - an strukturellen Maßnahmen, die Lehrende entlasten, damit diese sich mit den neuen Aufgaben vertraut machen und diese bewältigen können. Wieder einmal entsteht bei Lehrenden der Eindruck, „von höherer Stelle“ eine Reform verordnet $\mathrm{zu}$ kriegen und wieder einmal soll alles anders und besser werden, aber bitte ohne die alten Zöpfe (Verpflichtungen, Vorgaben, Bildungsinhalte usw.) zu kappen. Das ist bereits ein Fluch, der über der Umsetzung der Kompetenzorientierung liegt.

Ein Segen der Kompetenzorientierung für den Mathematikunterricht ist, dass sich der Fokus im Unterricht auf Anwendung und den Erwerb mathematischer Fertigkeiten und Expertise verschiebt, und dass Lernende bereits im Mathematikunterricht selbst Gelegenheit haben, in einem Teilgebiet kompetent $\mathrm{zu}$ werden. Wo Lernende früher darauf vertröstet wurden, dass sich die Anwendung ihres mathematischen Wissens später außerhalb der Schule finden würde („Grau ist alle Theorie und grün des Lebens goldner Baum"), fordert kompetenzorientierter Mathematikunterricht, das Leben entweder in den Unterricht zu bringen oder den Mathematikunterricht über die Schule hinaus auszudehnen und das Leben durch die mathematische Brille zu erforschen. Die damit einhergehende Differenzierung und Öffnung von Schule und Mathematikunterricht ist ein Segen - für alle Beteiligten.

Schließlich müssen sich Mathematiklehrende heute eingehender als vormals fragen, was in der Mathematikstunde, der Algebravorlesung und im Stochastikseminars tatsächlich gelehrt und was gelernt wurde. Dabei genügt es nicht, mathematisches Wissen in einen Trichter zu schütten in der Annahme, dass „wenn ich gelehrt habe, haben Schüler und Studenten auch gelernt". Kompetenzorientierung im Mathematikunterricht bedeutet eher das Gegenteil: „Ich habe erst dann gelehrt, wenn die SchülerInnen gelernt haben“. Das dürfte vor allem für Gymnasien und Universitäten eine ernstzunehmende Botschaft sein.

Es bleibt somit spannend, was die Erträge kompetenzorientierten Mathematikunterrichts sein werden. Was davon Fluch oder Segen ist, das wird spätestens fassbar, wenn die Kompetenzorientierung von der nächsten Reform abgelöst werden wird.

Prof. Dr. Brigitte Lutz-Westphal, Didaktik der Mathematik, Fachbereich Mathematik und Informatik, Freie Universität Berlin, Arnimallee 3, 14195 Berlin.brigitte.lutz-westphal@math.fu-berlin.de

\section{Aufruf zur Benennung von Kandidatinnen und Kandidaten für die Präsidiumswahlen 2018}

Im Auftrag des Präsidenten teile ich Ihnen mit, dass die Amtszeiten von fünf Präsidiumsmitgliedern in diesem Jahr auslaufen. Es handelt sich um folgende Positionen:

○ Präsident/Präsidentin und

- Vizepräsident/Vizepräsidentin.

Diese werden vom Präsidium für zwei Jahre gewählt.

- Schriftführer/Schriftführerin,

○ zwei weitere freie Sitze im Präsidium.

Diese werden von allen DMV-Mitgliedern für vier Jahre gewählt.

Die Positionen Präsident/in, Vizepräsident/in und Schriftführer/in beinhalten einen Sitz im Vorstand.
Nach § 2a der Wahlordnung ist das Präsidium verpflichtet, für jede frei werdende Position im Präsidium wenigstens einen Kandidaten/eine Kandidatin zu benennen. Weiterhin können andere Mitglieder Wahlvorschläge machen. Diese müssen nach $\S 2$ b der Wahlordnung von mindestens zehn Mitgliedern unterstützt werden und bis zum 30. April 2018 bei der Geschäftsstelle eingegangen sein.

Ich möchte alle Mitglieder nachdrücklich bitten, Vorschläge zu machen. Es wäre wünschenswert, mehr Kandidaten als Positionen zur Auswahl zu haben.

Prof. Dr. Daniel Grieser (Schriftführer der DMV) 